\title{
Measurement of Dynamical Density Profiles Using a Microwave Frequency Comb Reflectometer
}

\author{
Shigeru INAGAKI ${ }^{1,2)}$, Kimitaka ITOH ${ }^{2,3)}$, Takuma YAMADA ${ }^{2,4)}$, Sanae-I. ITOH ${ }^{1,2)}$, \\ Tokihiko TOKUZAWA ${ }^{1,3)}$, Akihide FUJISAWA ${ }^{1,2)}$, Naohiro KASUYA ${ }^{1,2)}$, Makoto SASAKI ${ }^{1,2)}$, \\ Yoshihiko NAGASHIMA ${ }^{1,2)}$ and Hiroyuki ARAKAWA ${ }^{5)}$ \\ ${ }^{1)}$ Research Institute for Applied Mechanics, Kyushu University, Kasuga, Fukuoka 816-8580, Japan \\ ${ }^{2)}$ Itoh Research Center for Plasma Turbulence, Kyushu University, Kasuga, Fukuoka 816-8580, Japan \\ ${ }^{3)}$ National Institute for Fusion Science, Toki, Gifu 509-5292, Japan \\ ${ }^{4)}$ Faculty of Arts and Science, Kyushu University, Nishi-ku, Fukuoka 819-0395, Japan \\ 5) Japan Atomic Energy Agency, Naka, Ibaraki 311-0193 Japan
}

(Received 26 July 2013 / Accepted 27 September 2013)

\begin{abstract}
We propose the use of a microwave comb for measuring the density profile as a continuous function of radius with high temporal resolution. The analysis focuses on phase-sensitive convolution of the reflected waves: convolution of pulses can eliminate statistical noise. The time resolution for constructing the density profile is also discussed.
\end{abstract}

(c) 2013 The Japan Society of Plasma Science and Nuclear Fusion Research

Keywords: dynamical density profile, microwave frequency comb reflectometer, multiple scale turbulence, statistical variation of gradient

DOI: $10.1585 /$ pfr.8.1201171

\section{Introduction}

Recently, a statistical interpretation of plasma turbulence and transport has been developed [1]. For example, inhomogeneities in the mean plasma parameter are neither stationary nor smooth, but large-amplitude corrugations in the profile evolve dynamically [2-4]. This new area of experimental study, combined with studies of microscopic, mesoscopic, and macroscopic perturbations [5,6], will provide a new arena for the study of turbulent transport. To observe such corrugations, which can be essential to the dynamical response of turbulent plasmas, it is necessary to simultaneously measure the mean profile and its fluctuations as continuous functions of the radius.

For this challenging problem, we propose employing a microwave frequency comb reflectometer. Experimental tests are planned on the PANTA device [7]. Statistical noise can be eliminated by convoluting pulses. The time resolution for constructing the density profile is found here to be on the order of $1 \mu \mathrm{s}$. This experimental method is very promising for developing the physics of plasma turbulence and transport.

\section{Model}

To illustrate our proposed approach, we consider a one-dimensional microwave reflection of the O-mode. The reflected wave has a frequency-dependent delay time $\tau(\omega)$ relative to the injected wave. This delay corresponds to the distance between the reference position and the location of the reflection, $x_{\text {ref }}(\omega)$, which is determined by the condition $\omega=\omega_{\mathrm{p}}(n(x))$ for the O-mode wave, where $n(x)$ is the density profile. The incident wave is expressed as

$$
\phi^{\text {in }}(t)=\int \mathrm{d} \omega \exp (-i \omega t) F(\omega),
$$

where $F$ is a weighting function that determines the envelope of the wave packet. For a microwave frequency comb, the spectrum is given by a Fourier series, but for simplicity we write it here in the form of Eq. (1). The phase of the carrier wave is precisely controlled with respect to the shape of the envelope (Fig. 1 (a)). In the simplified limit of geometrical optics, the reflected field is modified by the dispersion introduced by inhomogeneities in the density profile:

$$
\phi^{\mathrm{ref}}(t)=\int \mathrm{d} \omega \exp (-i \omega(t-\tau(\omega))) F(\omega) .
$$

Figure 1 (b) illustrates a schematic of a reflected wave. In principle, the amplitude of each Fourier component $F$ can
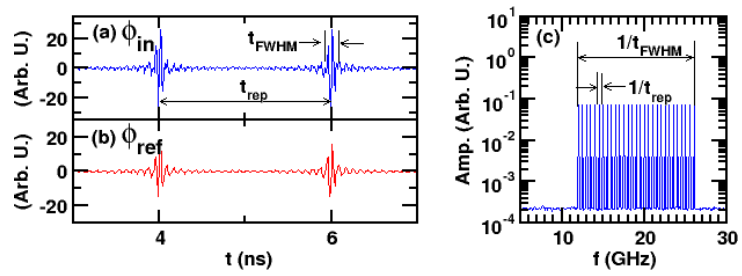

Fig. 1 Temporal evolution of (a) incident and (b) reflected microwaves and (c) the Fourier spectrum of the incident wave. 


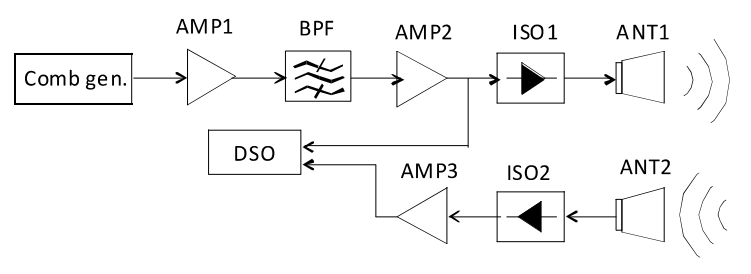

Fig. 2 Block diagram of the system.

also be modified owing to absorption by the plasma and other reasons. This issue is discussed later, and here we leave $F$ unchanged. Comparing $\phi^{\text {in }}$ and $\phi^{\text {ref }}$, the delay as a function of frequency, $\tau(\omega)$, is determined immediately. The delay $\tau(\omega)$ is obtained as a continuous function of the frequency. That is, the density profile is given as a continuous function of the position, $n(x)$.

\section{Experimental Setup}

Such a phase-sensitive convolution is possible and is currently being tested experimentally. Figure 2 illustrates the circuit used in the observations. The incident waveform is illustrated, i.e., the full width at half maximum of the envelope is $0.07 \mathrm{~ns}$ (corresponding to a frequency range of $12-27 \mathrm{GHz}$ ), and the repetition period is $2 \mathrm{~ns}$ (corresponding to a repetition frequency of $0.5 \mathrm{GHz}$ ). The reflected wave is received, and the incident and reflected wave signals are directly transferred to the digital storage oscilloscope (DSO), which has a frequency band of $33 \mathrm{GHz}$ (the sampling frequency is $80 \mathrm{GHz}$ ), so the waveforms of the incident and reflected signals are detected in the form of digital signals with very high temporal resolution. Our system enables simultaneous monitoring of fluctuation levels at more than 20 distinct spatial locations with very high temporal resolution (eight channels in previous work [8]).

Convolution of pulses allows elimination of noise. For testing purposes, a smooth and linearly increasing density profile was chosen as the target. Random noise signals having relative amplitudes of $5 \%$ and $10 \%$ of the signal were superimposed on the incident and reflected waves, respectively. The result of this example is shown in Fig. 3. Convolution with five pulses (i.e., measurement time window of $10 \mathrm{~ns}$ ) gave the result shown by the dotted line. The corrugations are the effects of the noise. By convolution with $N$ pulses, the influence of the noise was confirmed to decrease as $1 / N^{1 / 2}$. Convolution of 500 pulses (i.e., measurement time window of $1 \mu \mathrm{s}$ ), eliminated the errors caused by the noise, as shown by the solid line. This result indicates that the density profile can be constructed as a continuous function of radius with a time resolution of $1 \mu \mathrm{s}$.
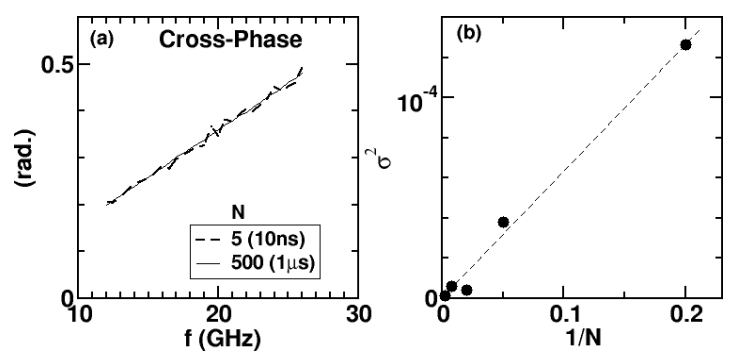

Fig. 3 (a) Reconstructed phase-delay structure and (b) convergence of variance in the phase.

\section{Summary}

We have shown that by using a microwave frequency comb, the density profile can be constructed as a continuous function of radius with a time resolution of $1 \mu \mathrm{s}$. This method will be useful in developing modern views of turbulence and transport based on nonlinear, nonlocal, and statistical interpretations.

Note that the laser comb and the optical frequency comb interferometer, could also be used in advanced plasma diagnostics [9]. If the amplitude of each Fourier component is modified in the reflected wave as $F^{*}$, then $F$ in Eq. (2) is replaced by $F^{*}$. When the attenuation is only in the reduction of the amplitude, this change does not influence the determination of the profile. In general cases, like mode conversion, the phase could also be influenced by the attenuation. These processes may limit the applicability of this method and will be discussed in future work.

This work is partly supported by the Grant-in-Aid for Scientific Research of JSPF, Japan (21224014, 23244113, 23360414) and by the collaboration programs of NIFS (NIFS13KOCT001) and of the RIAM of Kyushu University and Asada Science Foundation.

[1] S.-I. Itoh, J. Plasma Fusion Res. 86, 334 (2010).

[2] T.S. Hahm et al., Plasma Phys. Control. Fusion 46, A323 (2004).

[3] X. Garbet et al., Phys. Plasmas 14, 122305 (2007).

[4] S. Sugita et al., Plasma Phys. Control. Fusion 54, 125001 (2012).

[5] P.H. Diamond et al., Plasma Phys. Control. Fusion 47, R35 (2005).

[6] A. Fujisawa, Nucl. Fusion 49, 013001 (2009).

[7] S. Oldenbürger et al., Plasma Phys. Control. Fusion 54, 055002 (2012).

[8] W.A. Peebles et al., Rev. Sci. Instrum. 81, 10D902 (2010).

[9] H. Arakawa et al., presented at Jpn. Soc. Plasma and Fusion Research (2012, Kasuga) 29D09P. 